\title{
Transgenerational response to nutrition, early life circumstances and longevity
}

\author{
Gunnar Kaati $^{\star, 1,2}$, Lars Olov Bygren ${ }^{2,3}$, Marcus Pembrey ${ }^{4}$ and Michael Sjöström ${ }^{2}$
}

${ }^{1}$ Department of Public Health and Clinical Medicine, Umea University, Umeå, Sweden; ${ }^{2}$ Department of Biosciences and Nutrition, Karolinska Institutet, Stockholm, Sweden; ${ }^{3}$ Department of Community Medicine and Rehabilitation, Umea University, Umeå, Sweden; ${ }^{4}$ Clinical and Molecular Genetics Unit, Institute of Child Health, University College,

London, UK

Nutrition might induce, at some loci, epigenetic or other changes that could be transmitted to the next generation impacting on health. The slow growth period (SGP) before the prepubertal peak in growth velocity has emerged as a sensitive period where different food availability is followed by different transgenerational response (TGR). The aim of this study is to investigate to what extent the probands own childhood circumstances are in fact the determinants of the findings. In the analysis, data from three random samples, comprising 271 probands and their 1626 parents and grandparents, left after exclusions because of missing data, were utilized. The availability of food during any given year was classified based on regional statistics. The ancestors' SGP was set at the ages of 8-12 years and the availability of food during these years classified as good, intermediate or poor. The probands' childhood circumstances were defined by the father's ownership of land, the number of siblings and order in the sibship, the death of parents and the parents' level of literacy. An earlier finding of a sex-specific influence from the ancestors' nutrition during the SGP, going from the paternal grandmother to the female proband and from the paternal grandfather to the male proband, was confirmed. In addition, a response from father to son emerged when childhood social circumstances of the son were accounted for. Early social circumstances influenced longevity for the male proband. TGRs to ancestors' nutrition prevailed as the main influence on longevity.

European Journal of Human Genetics (2007) 15, 784-790; doi:10.1038/sj.ejhg.5201832; published online 25 April 2007

Keywords: early life; social environment; food availability; sex-specific transgenerational responses; mortality

Introduction

There seem to be transgenerational responses (TGRs) to variable food availability during the slow growth period (SGP), before prepubertal peak in the growth velocity during the childhood. We have earlier suggested that such

*Correspondence: Dr G Kaati, Department of Public Health and Clinical Medicine, Umeå University, SE-90541 Umeå, Sweden.

Tel: + 4690 7851212; Fax: + 4690 137919;

E-mail: Gunnar.Kaati@socmed.umu.se

Received 16 January 2007; revised 16 March 2007; accepted 17 March 2007; published online 25 April 2007 exposures give rise to responses, possibly in gene expression, in the next generations. ${ }^{1-2}$ However, an alternative explanation is that genetic selection through differential survival to maturity and/or the proband's own early life experiences $^{3-5}$ is the main determinant and not TGRs, such as epigenetic inheritance. (The term 'proband' is used for the index case, drawn at random from birth cohorts, not ascertained on disease status; an individual being studied.)

Nutritional change during the embryonic and fetal life, thought to change the placental size, is followed by doubling of the adult stroke mortality. ${ }^{6}$ Genes, such as 
those controlling placental size were known to be apt to genomic imprinting, ${ }^{7}$ and epigenetic control was thus one possible explanation.

TGRs from a mother's nutrition during her childhood can influence her child's risk as an adult for cardiovascular disease, diabetes mellitus and hypertension. ${ }^{8}$ The nutrition of the grandmother during pregnancy does not only influence the mother's nutrition during her fetal life but also the grandchild's birth weight. ${ }^{9}$ This led to an exploration of nutritional influences further back in the family lineage. The first male line TGRs to food supply in humans to be reported was our finding that the longevity was reduced for descendants following 'overeating' among boys during the SGP in the grandparental generation. ${ }^{1}$ The effect was found to relate to cardiovascular disease and diabetes. ${ }^{2}$ Now there seems to exist a maleline TGR in humans linking paternal grandparental nutrition to transgenerational mortality in a sex-specific manner. ${ }^{10}$ Further reports of TGRs in humans are few. They include a TGR to paternal mid-childhood tobacco smoking influencing early growth in sons, ${ }^{10}$ and paternal betel nut chewing linked to early onset of the metabolic syndrome in offspring. ${ }^{11}$

In explaining these observations, epigenetic inheritance has to be considered as a possible mechanism, being particularly visible when transmission is down the male line. ${ }^{12-13}$ Epigenetics refers to heritable changes in gene function that cannot be explained by changes in DNA sequence, with DNA methylation patterns being an important contribution to epigenetic memory during development. ${ }^{14}$ We have suggested the $\mathrm{X}$ and $\mathrm{Y}$ chromosomes could be involved in these sex-specific transgenerational effects. ${ }^{10}$ However, the observed transgenerational effects could be mediated by mechanisms other than epigenetic inheritance, for example by responsive DNA changes such as DNA amplification or change in telomere length. ${ }^{15,} 16$ The aim of this study was to consider to what extent the longevity of the probands are in fact determined by their own childhood circumstances rather than TGRs.

\section{Materials and methods}

The probands numbering 319 individuals were drawn as a random 50\% sample of those born 1890, 1905 and 1920 in Överkalix, Sweden. Excluded were those not traced, probands with missing data on the childhood circumstances and those whose ancestors' birth dates were unknown leaving 271 (88\%) individuals and their 1626 parents and grandparents for the analysis. ${ }^{2}$ Owing to the labour-intensive nature of the data collection, it was not possible to include all births in the cohorts years.

Childhood circumstances were defined by seven variables (landownership, parental deaths during the pro- bands' childhood up to 12 years, parents' literacy according to the clergy, the number of siblings and the order in the sibship).

Food availability in the province including the research area was determined from official statistics for the years $1803-1815$ and $1850-1900^{17}$ and the estimates of a 19 th century statistician $^{18}$ for the years $1816-1849$. The availability was classified into good, intermediate and poor based on his six-grade scale. The classification has been described elsewhere. ${ }^{1}$

Growth velocity during two childhood periods, the prepubertal peak and the SGP before the peak, was defined out of a current child growth velocity curve ${ }^{19}$ and the 19th century ages of puberty for boys and girls. ${ }^{20}$ The SGP was set at the age of 8-11 years for female ancestors and at 9-12 years for male ancestors.

\section{Statistical analysis}

Proportional hazards analyses of the mortality of the male and female probands, presented as hazard ratios (HR), ${ }^{21,22}$ were performed separately. Independent variables were nine variables determining the probands' childhood circumstances, and ancestors' nutrition during their SGP. The model is based on hypothesis of the causal mechanisms. Accordingly, independent variables were introduced in a logical manner beginning with the parental influences, then adding the social circumstances starting with landownership. Sensitivity analysis for consanguinity was performed by randomly withdrawing one cousin if more than one and rerun of the analyses. The data analysis for this paper was generated using SAS software. ${ }^{23}$

\section{Results}

The ancestors' nutrition

Apart from the poor soil that the majority of the farmers in the study area had to contend with, the food supply was also often poor during the 19th century owing to the failures of crops. During the century, the parish experienced 20 years of good availability of food and 21 years of poor availability according to our classification, and 59 years of moderate availability of food.

The harvests of 1800, 1812, 1821 and 1829 were classified as total crop failures. This was also true of 1809 because of the Swedish-Russian war when the armies laid their hands on much of the local stocks and made it difficult for the harvest to be brought in. In the period 1829-1837, all years were years of total crop failures. The severity of these years is conveyed by the parish clergyman's notes in the church registry covering 1836 that 'nothing exceptional is to remark but that this year was the seventh year of failure of crops and the most severe and pressing of them all.

In 1837, he notes that 'nothing exceptional to remark but that the eighth year of crop failure occurred, more 
severe than the foregoing'. In 1851 and 1856, there were again total crop failures and the harvests in 1867, 1877, 1881, 1888 and 1889 were poor. A surfeit of food was available after the harvest in 1799, 1801, 1813-1815, 1822, $1825-26,1828,1841,1844,1846,1853,1860-61,1863$, $1870,1876,1879$ and 1880. All other years had moderate harvests, neither very poor nor good. Of the paternal grandmothers in the cohorts 39\% had experienced famine during their SGP, 50\% of the paternal grandfathers had had the same experience. Good harvests during the SGP were experienced by 46 and 47\%, respectively.

\section{The empirical distribution of longevity}

The non-adjusted mortality hazards among probands, whose paternal grandparents had experienced at least 1 year of good availability during their SGP, revealed more premature deaths among them compared with those probands where paternal grandparents had not had any such good year during their SGP. A marked sex-linked influence could be seen (Figure 1).

\section{The social contexts and life circumstances of the probands}

About half of the fathers of the probands owned land, which in this parish usually meant that they lived on units with 1-2 Ha (around 2 acres) of arable land. The most affluent of the farmers had holdings that were no larger than $10 \mathrm{Ha}$ (24 acres), and they were very few. ${ }^{24}$ Almost half of the probands fathers and mothers were farmhands and maidens.

Six per cent of the fathers and $10 \%$ of the mothers died during the probands' childhood up to their 13th birthday.

The families were generally large and $24 \%$ of the probands had 10 or more siblings (one had a sibship of 15). Seventy per cent of the probands had 5-9 siblings. The average size of the shipship was eight children. The parents' literacy as youngsters was rarely to the satisfaction at least of the clergy as only $22 \%$ of the fathers and $24 \%$ of the mothers had passed their literacy test (Table 1).

\section{TGR to nutrition taking account of the probands' early social circumstances}

The male probands had an increased risk of mortality if their fathers had good nutrition during their SGP. This association emerged as significant when the proband's childhood circumstances were taken into account. The association of the paternal grandfathers food availability during the SGP on the grandson's hazards ratio (HR) and paternal grandmother's food availability on the granddaughter's HR was less pronounced after the introduction of the probands' childhood circumstances. Good nutrition in the ancestor is associated with poor survival of the proband.

The childhood environment had influences on the male probands HR. For them, the mother's death during the childhood was detrimental. Conversely good literacy of the

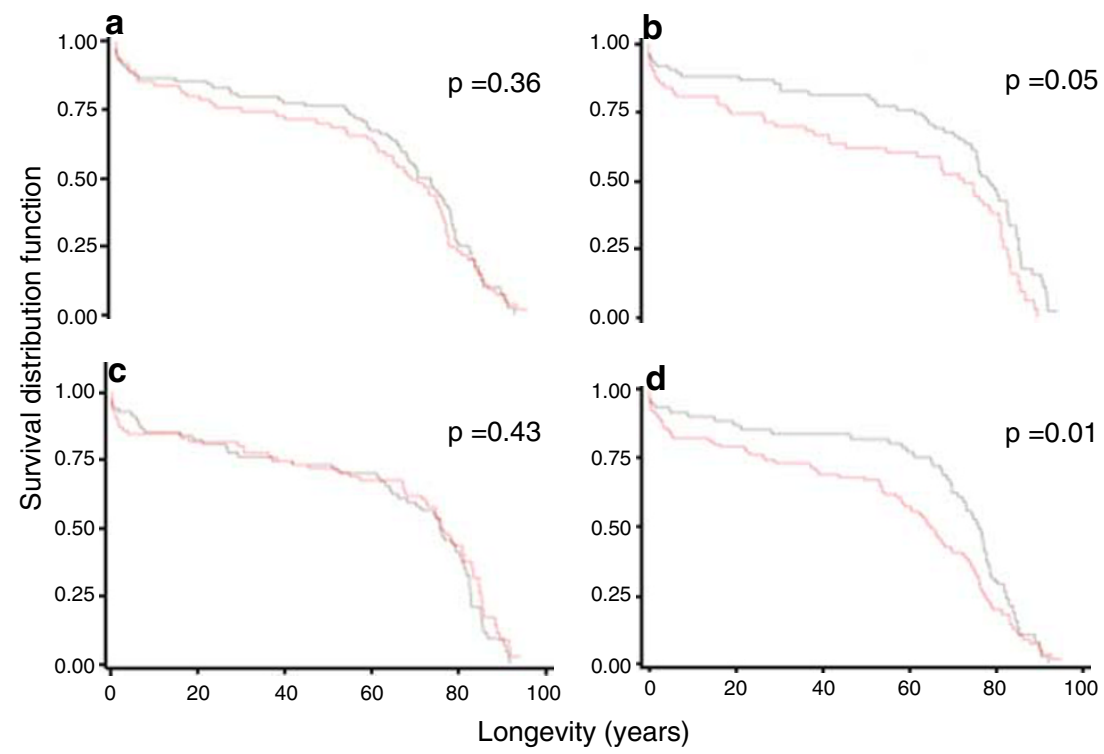

Figure 1 The empirical distribution of longevity. Male and female probands survival when the paternal grandparent experienced at least 1 year of good availability of food in the environment (dashed line), or no year of good availability (solid line), during their SGP that is at $8-10$ years of age for the paternal grandmother and at 9-12 years of age for the paternal grandfather. No adjustments. $N=306$. (a) Paternal grandmothers availability of food during her SGP and the survival of the male probands. (b) Paternal grandmothers availability of food during her SGP and the survival of the female probands. (c) Paternal grandfathers availability of food during his SGP and the survival of the female probands. (d) Paternal grandfathers availability of food during his SGP and the survival of the male probands. 
Table 1 The sample of probands by independent variables and sex

\begin{tabular}{lccc}
\hline & $\begin{array}{c}\text { Male } \\
\mathrm{N}=164\end{array}$ & $\begin{array}{c}\text { Female } \\
\mathrm{N}=139\end{array}$ & $\begin{array}{c}\text { Both sexes } \\
\mathrm{N}=303\end{array}$ \\
\hline Father landowner $^{\mathrm{a}}$ & 86 & 73 & 159 \\
Father without land $^{\text {Father died }}$ & 78 & 66 & 144 \\
Father alive $^{\mathrm{b}}$ & 13 & 5 & 18 \\
Mother died $^{\mathrm{b}}$ & 151 & 134 & 285 \\
Mother alive & 15 & 14 & 29 \\
Proband firstborn & 149 & 125 & 274 \\
Proband lastborn & 23 & 23 & 46 \\
Proband middle-born & 27 & 16 & 43 \\
& 110 & 97 & 207 \\
Number of siblings & & & \\
0-4 & 27 & 19 & 46 \\
5-9 & 97 & 86 & 183 \\
10-14 & 39 & 33 & 72 \\
15 & 1 & 1 & 2 \\
The literacy level & & & \\
Father & & & \\
$\quad$ Pass & 113 & 113 & 226 \\
$\quad$ Fail & 41 & 22 & 63 \\
Mother & & & \\
$\quad$ Pass & & 102 & 222 \\
Fail & 40 & 30 & 70 \\
\hline
\end{tabular}

aLandowner equals both those with farms and smallholdings.

${ }^{\mathrm{b}}$ The mother/father died before the proband was 13 years of age.

mother and possibly the father's ownership of land protected the male proband. For the female proband, no such influence of the childhood environment could be detected (Table 2).

\section{Population genetics considerations}

Consanguinity was considered. None of the probands, for instance, who died of diabetes had the same paternal grandfather but some of the non-diabetic probands had. A sensitivity analysis, including just one male randomly drawn proband with the same paternal grandfather $(n=143)$, did not change the TGR of diabetic mortality HR from ancestors food availability during their SGP. The HR if the paternal grandfather had experienced some year(s) of plenty during his SGP changed from 1.45 to 1.50 , the $P$-values from 0.049 to 0.047 .

Correspondingly, his experiencing year(s) of poor food availability during his SGP, the HRs of the male proband changed from 0.50 to 0.64 and the $P$-values from 0.006 to 0.023 .

\section{Discussion}

Genetic selection across the generations was considered in the earlier analysis, ${ }^{2}$ and signs of selection among ancestors experiencing different food availability during their SGP were not detected. In the cohorts, selection might have resulted in similar age at death combined with diminishing variations around the average over the generations. This was not, however, the case as the average age at death and the variations around it were similar across the generations. A high infant mortality did not vary with difference in food availability during the SGP.

The results provide further support for the hypothesis that a sex-specific, male-line TGR-system exists in humans, capturing nutritionally related information from the previous generation(s).

The research area was colonized in the medieval time. The parish became very homogeneous in terms of population, manifested, for example, by the particular variant of the Swedish language spoken there. It can be seen as an 'island' surrounded by Sami people and communities to the north and west and Finnish speaking people to the east, dividing hamlets or villages just a couple of kilometres from each other along immutable language or cultural lines. Specific recessive monogenic diseases can emerge in such populations owing to the chance genetic makeup of the small founder population and subsequent inbreeding. In Överkalix, there are currently cases of infantile genetic agranulocytosis $^{25}$ and Gaucher's disease. ${ }^{26}$ However, monogenic diseases are not more common than elsewhere in the province. ${ }^{27}$ The multifactorial disease multiple sclerosis, is common in one family in Överkalix. ${ }^{28}$

In an earlier paper, we considered the possibility of genetic selection of an allele for infant survival and diabetes susceptibility for example, but no evidence of selection through differential infant mortality among ancestors with different availability of food during their SGP could be found. ${ }^{2}$ Here we show, using sensitivity analysis, that genetic relatedness does not account for the transgenerational effects observed, so enrichment of the diabetic probands with multiple descendants from one ancestor who happened, by chance, to transmit a susceptibility allele is not an explanation.

Control of some of the probands' own early social life circumstances did not change our interpretation of the early findings. Instead, the early hypotheses of a father to son influence only emerged after this control, indicating that it had been confounded by the probands' social circumstances during their childhood. Some of the decrease in power can be explained by a decrease in the number of probands in this analysis from 164 to 146 male probands and from 139 to 125 female probands owing to missing data. The social context exerted influence but the transgenerational associations remained or emerged, indicating that sperm carry information about the ancestral nutrition. The mother being literate and alive had a demonstrable bearing on the survival of male probands, but not on the female probands.

There are several inherent problems with this type of study. The sample size was necessarily limited by the work involved in tracing historical data. We have only limited 
Table 2 The proportional HR in longevity between probands, whose ancestors experienced at least 1 year of good availability of food or poor availability of food during their SGP, whose fathers own land or not, whose parents died or not, who was first, middle or last born, whose sibship size varied and whose parents varied in literacy.

\begin{tabular}{|c|c|c|c|c|}
\hline & \multicolumn{2}{|c|}{ Male probands } & \multicolumn{2}{|c|}{ Female probands } \\
\hline & \multicolumn{2}{|l|}{$N=146$} & \multicolumn{2}{|l|}{$\mathrm{N}=125$} \\
\hline & $H R$ & P-value & $H R$ & P-value \\
\hline \multicolumn{5}{|c|}{ Ancestors experienced good nutrition $(N=864)$ during the SGP } \\
\hline Mother & 1.05 & 0.82 & 1.50 & 0.10 \\
\hline Father & 1.70 & $0.01^{\mathrm{a}}$ & 1.14 & 0.60 \\
\hline Maternal grandmother & 0.92 & 0.70 & 0.70 & 0.14 \\
\hline Maternal grandfather & 0.77 & 0.19 & 1.18 & 0.51 \\
\hline \multicolumn{5}{|l|}{ The proband's childhood } \\
\hline Father landowner & 0.79 & 0.24 & 1.07 & 0.79 \\
\hline Father died before proband's 13th year & 0.92 & 0.80 & 1.03 & 0.96 \\
\hline Mother died before proband's 13 th year & 2.03 & 0.04 & 0.41 & 0.88 \\
\hline First born & 1.19 & 0.54 & 1.13 & 0.70 \\
\hline Last born & 1.08 & 0.77 & 1.26 & 0.51 \\
\hline \multicolumn{5}{|c|}{ Ancestor experienced poor nutrition $(N=537)$ during the SGP } \\
\hline Mother & 0.77 & 0.27 & 0.73 & 0.23 \\
\hline Father & 1.11 & 0.61 & 1.03 & 0.89 \\
\hline Maternal grandmother & 1.19 & 0.37 & 1.18 & 0.46 \\
\hline Maternal grandfather & 1.21 & 0.33 & 1.14 & 0.55 \\
\hline Paternal grandmother & 1.14 & 0.55 & 0.66 & $0.06^{\mathrm{d}}$ \\
\hline Paternal grandfather & 0.56 & $0.01^{\mathrm{e}}$ & 1.18 & 0.48 \\
\hline \multicolumn{5}{|l|}{ The proband's childhood } \\
\hline Father landowner & 0.70 & 0.07 & 0.96 & 0.87 \\
\hline Father died before proband's 13th year & 1.27 & 0.50 & 1.06 & 0.92 \\
\hline Mother died before proband's 13th year & 2.13 & 0.04 & 1.29 & 0.49 \\
\hline First born & 1.00 & 0.99 & 0.92 & 0.79 \\
\hline Last born & 1.22 & 0.44 & 1.35 & 0.39 \\
\hline
\end{tabular}

HR, hazards ratio; SGP, slow growth period.

a Before all male proband's childhood circumstances were introduced: $n=146, \mathrm{HR}=1.32, P=0.13$.

${ }^{\mathrm{b}}$ Before all female proband's childhood circumstances were introduced: $n=139, \mathrm{HR}=1.75, P=0.01$.

'Before all male proband's childhood circumstances were introduced: $n=164, \mathrm{HR}=1.45, P=0.05$.

'Before all female proband's childhood circumstances were introduced: $n=135, \mathrm{HR}=0.71, P=0.01$.

'Before all male proband's childhood circumstances were introduced: $n=164, \mathrm{HR}=0.60, P=0.01$.

knowledge about the proband's adult life and even less about the ancestors. Food availability has to be used as a surrogate for actual nutrition. In this analysis, the problem of multiple comparisons or multiplicity had to be faced. ${ }^{29}$ Although it is possible to safeguard for mass significance, at least to some extent, the necessity of such safeguards can be discussed, but it is of course necessary to be aware of the increased risk of errors in such analysis. ${ }^{30,31}$ In this study, the problem of multiplicity is less serious, as few variables were tested and all were prior hypotheses. Furthermore, in this case the pattern of results is biologically plausible and conforms to the hypotheses.
The influence of nutrition emerged in spit of possible misclassification of exposures and strengthens the support of the results. Misclassification of exposures to famines and abundance is probably negligible, as the estimates are based on several independent sources. On the other hand, misclassification of individuals possibly exists although they ought to be few considering the narrow span of the social structure in the study area. Still some real influences ought have been missed.

The transgenerational effects seem robust, but the nature of the triggering exposure is unknown. It may be the food intake itself or something strongly associated with food 
availability. Nevertheless, the sex-specific nature of the TGRs with the paternal grandfather's food supply having no effect on the mortality of granddaughters and the paternal grandmother's food supply having no effect on grandsons serves as an internal control. ${ }^{10}$ Social patterning and learning down the generations of the male line is, therefore, unlikely to account for much, if any, of the observed transgenerational effects and a more direct biological explanation needs to be sought. Our results strengthen the theory of a transgenerational mechanism, which appears to capture nutritional information in a sensitive period of mid-childhood from the previous generation(s). This requires a mechanism for transmitting environmental exposure information that then alters gene expression in the next generation(s). A TGR down the male line implies the ancestral exposure information is carried by the sperm.

Epigenetic inheritance is a strong candidate for three reasons; some epigenetic states can be transmitted to the next generation(s), specific nutritional exposures can alter the epigenetic state and TGRs to specific exposures have been shown in experimental animals and humans. However, examples that integrates all three elements are only just emerging. ${ }^{32-35}$ Genomic imprinting establishes the principle of transgenerational epigenetic inheritance with an imprint tag or epigenetic mark placed in one generation influencing gene expression in the next. ${ }^{36}$ Murine studies have demonstrated transgenerational inheritance of epigenetic states at the agouti-viable yellow $\left(\mathrm{A}^{\mathrm{vy}}\right)$ allele and the Axin-fused (Axin ${ }^{F u}$ ) allele, the latter through both maternal and paternal transmission. ${ }^{37}$ In these transgenerational studies, the particular epigenetic states arise stochastically, being metastable epialleles, ${ }^{38}$ and were not induced by a particular exposure. Interestingly though, the DNA methylation status, gene expression and phenotype related to both these gene loci can be modified by dietary methyl donor supplementation in early development. ${ }^{39,} 40$ There is a recent report that nutritional supplementation can lead to germline epigenetic modification in agoutimouse model. ${ }^{35}$

The three reports of TGRs in humans show some concordance in outcome in the descendants. ${ }^{1,2,10}$ The Överkalix studies demonstrate effects of ancestral food supply on cardiovascular and diabetic deaths, ${ }^{2}$ the ALSPAC study shows paternal mid-childhood smoking is linked to a greater body mass index at 9 years in sons, ${ }^{10}$ and a study in Taiwan shows paternal betel nut chewing is associated with early onset of the metabolic syndrome in offspring. ${ }^{11}$ The last study follows on from work in mice that found that betel feed induced an increase in hyperglycaemia, central obesity and islet enlargement. Males mated with controls 4 weeks after 6 days of betel nut feed produced similar features of the metabolic syndrome in 3-10\% of subsequent (F1-F3) non-betel fed generations. ${ }^{41}$
None of these human studies provides any data on the mediating molecular mechanism. There is evidence (see above) of epigenetic inheritance starting to emerge in the agouti mouse model. ${ }^{35,37,38}$ There is evidence in rats that the endocrine disruptor vinclozolin induces epigenetic (methylation) alterations in the male germ line that are transmitted to subsequent generations who then have a higher incidence of adult onset diseases. ${ }^{33-34}$ However, the causal link between the epigenetic changes and the diseases has yet to be established. A study of paramutation in mice, where the untransmitted mutant allele nevertheless causes phenotypic effects in subsequent generations, shows that this is mediated by the aberrant messenger RNA being packaged into the sperm and then disrupting the function of the normal gene in the next generation(s). ${ }^{42}$ In addition to these epigenetic mechanisms, it is theoretically possible that 'responsive' DNAsequences, ${ }^{15-16}$ viruses or prions ${ }^{43}$ could mediate the trans generational effects, although the sex-specific nature of the transmissions makes the latter two mechanisms unlikely.

Replication of the findings on other cohorts exposed to nutritional excesses or deficits are now required, as well as human and animal studies where the molecular mechanisms can be detected. The finding that the mother's literacy level influenced the proband's mortality was expected, but the observation that the influence was only found in the male proband warrants further study. Evidence that fetal and infant life is an exposure-sensitive period in females ${ }^{10}$ suggests that future studies should focus on early life exposure as well as the SGP.

The findings revive somewhat the evolutionary debate over the role of the environment, and its specific interplay with biological evolution. Our research suggests a closer degree of integration between environment and biological evolution. ${ }^{15,44}$

In conclusion, there were influences on the probands' longevity from their childhood circumstances. However, the observed TGR's on food availability during ancestor's SGP seemed to be the main mover in determining the probands longevity. The results provide further support for the hypothesis that a sex-specific, male-line TGR-system exists in humans, capturing nutritionally related information from the previous generation(s), resulting in differential mortality depending on ancestors' exposure during the SGP. The transgenerational effects remain after taking account of the genetic relatedness and early life circumstances of the probands.

\section{References}

1 Bygren LO, Kaati G, Edvinsson S: Longevity determined by ancestors' over-nutrition during their slow growth period. Acta Biotheoretica 2001; 49: 53-59.

2 Kaati G, Bygren LO, Edvinsson S: Cardiovascular and diabetes mortality determined by nutrition during parents' and grandparents' slow growth period. Eur J Hum Gen 2002; 10: 682-688. 
3 Drake AJ, Walker BR: Intergenerational effects of fetal programming: non-genomic mechanisms for the inheritance low birth weight and cardiovascular risk. J Endocrinology 2004; 180: 1-16.

4 Forsdahl A: Are poor living conditions in childhood and adolescents an important risk factor for arteriosclerotic heart disease? Br J Prev Soc Med. 1977; 31: 91-95.

5 Barker DJP, Osmond C: Infant mortality, childhood nutrition, and ischemic heart disease in England and Wales. Lancet 1986; 1: $1077-1081$.

6 Bygren LO, Edvinsson S, Broström G: Change in food availability during pregnancy: is it related to adult sudden death from cerebro- and cardiovascular disease in offspring. Am J Hum Biol 2000; 12: $447-453$.

7 Hall JG: Genomic imprinting: nature and clinical relevance. Ann Rev Med 1997; 48: 35-44.

8 Martyn CN, Barker DJ, Osmond C: Mothers' pelvic size, fetal growth, and death from stroke and coronary heart disease in men in the UK. Lancet 1996; 348: 1264-1268.

9 Lumey LH, Stein AD: Offspring birth weights after maternal intrauterine undernutrition: a comparison within sibships. Am J Epidemiol 1997; 146: 810-819.

10 Pembrey M, Bygren LO, Kaati G, et al., The ALSPAC Study Team: Sex-specific, sperm-mediated transgenerational respones in humans. effects. Eur J Hum Gen 2006; 14: 159-166.

11 Chen THH, Chiu YH, Boucher BJ: Transgenerational effects of betal quid chewing on the metabolic syndrome in the Keelung Community-based Integrated Screening Program. AM J Clin Nutr 2006; 83: 688-692.

12 Jablonka E, Lamb MJ: Epigenetic inheritance and evolution. Oxford: Oxford University Press, 1995.

13 Jablonka E, Lamb MJ: Evolution in four dimensions: genetic, epigenetic, behavioural and symbolic variation in the history of life. Cambridge Mass: MIT Press, 2005.

14 Bird A: DNA methylation patterns and epigenetic memory. Genes Dev 2002; 16: 6-21.

15 Yasui K, Mihara S, Zhao C et al: Alteration in copy numbers of genes as a mechanism for acquired drug resistance. Cancer Res 2004; 64: 1403-1410.

16 Epel ES, Blackburn EH, Lin J et al: Accelerated telomere shortening in response to life stress. Proc Natl Acad Sci USA 2004; 101: $17312-17315$

17 : Tabellverket 1749-1859. Umeå: Demographic Data Base, 2005.

18 Hellstenius J: Skördarna i Sverige och deras verkningar. (The harvests in Sweden and their consequences). Statistisk Tidskrift 1871; 77-119.

19 Prader A, Largo RH, Molinari L: Physical growth of Swiss children from birth to 20 years of age. First Zurich longitudinal study of growth and development. Helvetica Paediatrica Acta 1988; 43 (Suppl): 52.

20 Tanner JM: A history of the study of human growth. Cambridge: Cambridge University Press, 1981.

21 Andersen PK, Borgan Ö, Gill RD, Keiding N: Statistical models based on counting processes. New York: Springer, 1993.

22 Picton H, Briggs D, Gosden R: The molecular basis of oocyte growth and development. Mol Cell Endocrinol 1998; 145: 27-37.

23 SAS/STAT software: Version 8.2 of the SAS System for Windows. Cary, NC: SAS Institute Inc, 1999-2001.

24 Johansson F (red.) : Överkalix 1644-1944. Luleå: Luleå boktr, 1948.
25 Carlsson G, Fast A: Infantile genetic agranulocytos, morbus Kostman: presentation of six cases from the original 'Kostman family' and a review. Acta Paediatr 2001; 90: 757-764.

26 Dreborg S, Erikson A, Hagberg B: Gaucher Disease - Norrbottnian Type. I. General Clinical Description. Eur J Pediatr 1980; 133: 107-118.

27 Einarsdottir E: Mapping genetic diseases in northern Sweden. Umeå: Department of Medical Bioscienes Unit of Medical and Clinical Genetics, 2005.

28 He B, Giedraitis V, Ligers A et al: Sharing of a conserved haplotype suggests a susceptibility gene for multiple sclerosis at chromosome 17p11. Eur J Hum Genet 2002; 10: 271-275.

29 Senn SJ: Statistical issues in drug development. Chichester: Wiley, 1997.

30 Blom G, Holmquist B: Statistikteori Med Tillämpningar. Lund: Studentlitteratur, 1998.

31 Katz MH: Multivariable Analysis. Cambridge: Cambridge University Press, 1999.

32 Anway MD, Cupp AS, Uzumcu M, Skinner MK: Epigenetic transgenerational actions of endocrine disruptors and male fertility. Science 2005; 308: 1466-1469.

33 Chang HS, Anway MD, Rekow SS, Skinner MK: Transgenerational epigenetic imprinting of the male germline by endocrine disruptor exposure during gonadal sex determination. Endocrinology 2006; 147: 5524-5541.

34 Anway MD, Leathers C, Skinner MK: Endocrine disruptor vinclozolin induced epigenetic transgenerational adult onset disease. Endocrinology 2006; 147: 5515-5523.

35 Cropley JE, Suter CM, Beckman KB, Martin DIK: Germ-line epigenetic modification of the murine $\mathrm{A}^{v y}$ allele by nutritional supplementation. Proc Natl Acad Sci USA 2006; 103: $17308-17312$

36 Ferguson-Smith AC, Surani MA: Imprinting and the epigenetic asymmetry between parental genomes. Science 2001; 298: $1086-1089$.

37 Rakyan VK, Chong S, Champ ME et al: Transgenerational inheritance of epigenetic states at the murine $\mathrm{Axin}^{\mathrm{Fu}}$ allele occurs following maternal and paternal transmission. Proc Natl Acad Sci USA 2003; 100: 2538-2544.

38 Rakyan VK, Blewitt ME, Druker R, Preis JI, Whitelaw E: Metastable epialleles in mammals. Trends Genet 2002; 18: 348-351.

39 Waterland RA, Jirtle RL: Early nutrition, epigenetic changes at transposons and imprinted genes, and enhanced susceptibility to adult chronic diseases. Nutrition 2004; 20: 63-68.

40 Waterland RA, Dolinoy DC, Lin JR, Smith CA, Shi X, Tahiliani KG: Maternal methyl supplements increase offspring DNA methylation at Axin Fused. Genesis 2006; 44: 401-406.

41 Boucher BJ, Ewen SW, Stowers JM: Betl nut (Areca catechu) consumption and the induction of glucose intolerance in adult CD1 mice and in their F1 and F2 offspring. Diabetologia 1994; 37: $49-55$.

42 Rassoulzadegan M, Grandjean V, Gounon P, Vincent S, Gillot I, Cuzin F: RNA-mediated non-mendelian inheritance of an epigenetic change in the mouse. Nature 2006; 441: 469-474.

43 Shorter J, Lindquist S: Prions as adaptive conduits of memory and inheritance. Nat Rev Genet 2005; 6: 435-450.

44 Gould SJ: The structure of evolutionary theory. Cambridge: The Belknap Press of Harvard University Press, 2002. 